Ann. Sci.forest., I975, 32 (3), I69-I74.

NOTE TECHNIQUE

COMPARAISON

DE DEUX TECHNIQUES SPECTROMÉTRIQUES
D'ANALYSE DU CALCIUM DANS LES VÉGÉTAUX.
MISE EN ÉVIDENCE DE L'EFFET D'INTERFÉRENCE
DU PHOSPHORE SUR LES RÉSULTATS OBTENUS

A. CLÉmENT et C. NYS

Station de Recherches sur les Sols forestiers et la Fertitisation,

Centre National de Recherches forestieres, I. N.R. A.,

Champenoux, 54280 Seichamps

\title{
RÉSUMÉ
}

Les auteurs comparent les résultats de dosage du calcium dans les tissus foliaires obtenus par spectrophotométrie d'émission et spectrophotométrie d'absorption atomique.

L'interférence de l'ion phosphore en spectrophotométrie d'émission a été quantifié. Après correction des interférences par abaque dans le cadre de l'émission et par addition de chlorure de strontium pour l'absorption atomique, les deux méthodes apparaissent équivalentes.

\section{I. - INTRODUC'TION}

Ces dernières annéss l'analyse minérale a fait des progrès énormes grâce aux techniques d'analyses spectrométriques. Ces techniques, rappelons-le, utilisent certaines propriétés physiques des atomes métalliques soumis à diverses excitations.

La plus ancienne, la spectrométrie d'émission de flamme, a permis de simplifier considérablement les analyses des métaux alcalins et de certains alcalino-terreux tel le calcium. La plus récente, la spectrométric d'absorption atomique, est venue compléter la première de telle sorte qu'ensemble leur spectre d'activité couvre la quasi totalité des métaux. Ces méthodes complémentaires sont cependant susceptibles de se recouvrir au niveau de certains cations. Il est en effet possible d'identifier le calcium selon l'une ou l'autre de celles-ci avec des seuils de détections assez comparables. La solution sur laquelle le cation est analysé est un milieu anionique et cationique complexe, qui très souvent interfère sur le dosage de l'élément choisi. Nous étudierons plus spécialement l'interférence de l'ion $\mathrm{PO}_{4}^{--}$sur le dosage du calcium dans le cadre de la comparaison de l'analyse par émission de flamme et de l'analyse par absorption atomique. 


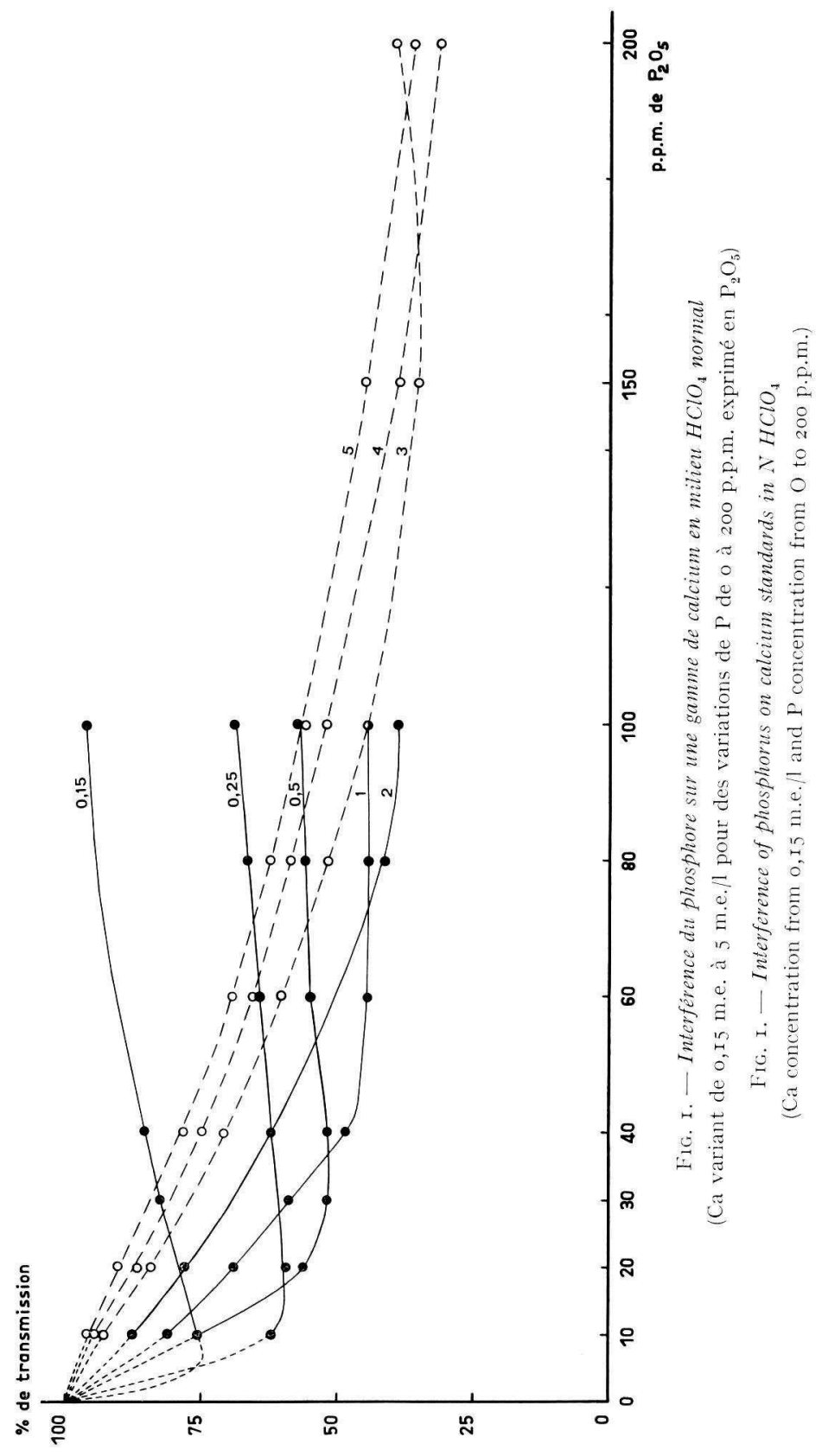




\section{II. - MATÉRIEL, E'T MÉTHODES}

Nous avons travaillé sur de la poudre d'aiguilles de résineux (Picea abies) et de feuilles vertes de feuillus (Fagus sylvatica, Populus nigra), séchées à l'étuve à $65^{\circ} \mathrm{C}$ pendant $4^{8}$ heures puis broyées. 99 échantillons ont été prélevés et analysés. La minéralisation est obtenue par une attaque humide en milieu acide perchlorique-eau oxygénée à chaud (CLÉMENT, I97 I) sur une masse connue d'échantillon. Le calcium contenu dans la solution d'attaque est dosé selon les deux méthodes.

\section{I. - Principe des méthodes utilisées}

Les deux méthodes s'appuient sur la loi de Kirchoff, selon laquelle tout élément chimique capable sous une excitation d'émettre une radiation caractéristique est également capable de l'absorber.

- En spectrométrie d'émission de flamme, le métal à analyser est soumis à une excitation thermique intense. Il émet une radiation caractéristique dont l'intensité est dans certaines conditions proportionnelle à sa concentration dans la solution.

- En spectrométrie d'absorption atomique, le métal à analyser est soumis à une radiation qui lui est caractéristique, c'est-à-dire à une radiation qu'il est susceptible d'absorber. La quantité de radiation qu'il absorbe est dans certaines conditions proportionnelle à sa concentration dans la solution.

$$
\text { 2. 2. - Protocoles analytiques }
$$

- Dosage par spectrophotométrie d'émission :

la solution est passée directement et l'échantillon est comparé à une gamme. Une correction de l'interférence du phosphore est effectuée à partir d'abaque (fig. I) ou de tableaux établis d'unc manière expérimentale.

- Dosage par spectrophotométrie d'absorption atomique :

Les interférences du calcium sont nombreuses principalement avec $\mathrm{SiO}_{3}, \mathrm{PO} \equiv, A 1$ et Fe (PINTA, I $97 \mathrm{I})$. Ces interactions sont corrigées par l'adjonction de lanthane ou de strontium. Nous effectuons le dosage après dilution de la solution d'attaque dans une solution de strontium. La solution finale contient environ I $500 \mu \mathrm{g}$ a de strontium.

\section{III. — RÉSULTATS}

Les résultats comparés sont ceux obtenus par émission après correction calculée de l'intertérence du phosphore avec ceux obtenus par absorption atomique, l'interférence étant dans ce cas corrigée par l'adjonction de chlorure de strontium. La différence entre les chiffres obtenus pour un même échantillon est due aux méthodes.

La tencur, en p. Ioo de calcium, de la matière sèche des échantillons varie de 0,20 à 2 p. IoO, ce qui englobe presque toujours les teneurs habituellement rencontrées dans les végétaux ligneux.

La figure 2 et le calcul statistique de comparaison de moyenne montrent que :

La différence des résultats pour un même échantillon est laible et inférieure aux erreurs qui peuvent être faites en cours de manipulation. La comparaison de moyenne de deux séries appariées par la méthode des couples montrent que les moyennes ne sont pas significativement différentes au risque de 5 p. Ioo. En effet :

$$
|\varepsilon|=\frac{m}{\mathrm{~S} / \sqrt{n}}=\mathrm{I}, 83 \quad(\text { avec } n=99)
$$

$$
t 5_{0}^{\circ}=\mathrm{I}, 96 \text { avec } m=-0,6_{+} \cdot \mathrm{IO}^{-2}\left(m=\text { moyenne de } x_{i}-y_{i}\right) \text {. }
$$


TABLEAU I

Résultats du dosage du calcium selon les deux méthodes

\begin{tabular}{|c|c|c|c|c|c|}
\hline No & $\underset{x}{\text { Entission }}$ & Ab. Atm. & No & $\begin{array}{c}\text { Enission } \\
x_{i}\end{array}$ & Ab. Atm \\
\hline 1 & 0,34 & 0,34 & 51 & 0,40 & 0,42 \\
\hline 2 & 0,33 & 0,33 & 52 & 0,22 & $0,2^{\prime} t$ \\
\hline 3 & 0,39 & 0,39 & 53 & 0,19 & 0,20 \\
\hline 4 & 0,33 & 0,31 & 54 & 0,25 & 0,26 \\
\hline 5 & 0,40 & 0,41 & 55 & 0,36 & 0,37 \\
\hline 6 & $0,3 /$ & $0,3 / 4$ & 56 & 0,29 & 0,34 \\
\hline 7 & 0,29 & 0,32 & 57 & 0,19 & 0,22 \\
\hline 8 & 0,25 & 0,32 & 58 & 0,36 & $0,3^{\prime}$ \\
\hline 9 & 0,36 & $0,{ }^{\prime}+0$ & 59 & 0,28 & 0,29 \\
\hline 10 & 0,21 & 0,21 & 60 & 0,34 & 0,32 \\
\hline 11 & 0,30 & 0,30 & 61 & 0,71 & 0,82 \\
\hline 12 & 0,31 & 0,32 & 62 & 0,36 & 0,35 \\
\hline 13 & 0,38 & 0,40 & 63 & 0,36 & $0,3^{\prime}$ \\
\hline $1^{\prime} \mathrm{k}$ & 0,38 & 0,40 & 64 & 2,25 & 2,36 \\
\hline 15 & 0,37 & 0,38 & 65 & 1,75 & 1,82 \\
\hline 16 & 0,25 & $0,0,1$ & 66 & 0,85 & 0,90 \\
\hline 17 & 0,25 & 0,26 & 67 & 0,72 & 0,72 \\
\hline 18 & 0,37 & 0,38 & 68 & 0,61 & 0,66 \\
\hline 19 & 0,37 & 0,38 & 69 & 0,76 & 0,78 \\
\hline 20 & 0,26 & 0,25 & 70 & 0,87 & 0,82 \\
\hline 21 & 0,38 & 0,38 & 71 & 0,70 & 0,74 \\
\hline 22 & 0,35 & 0,36 & 72 & 0,81 & 0,78 \\
\hline 23 & 0,27 & 0,29 & 73 & $0,3 / \mathbf{t}$ & 0,32 \\
\hline $2{ }^{\prime}$ & 0,20 & 0,21 & 74 & 0,26 & 0,22 \\
\hline 25 & $0,2 t$ & 0,21 & 75 & 0,12 & 0,12 \\
\hline 26 & 0,26 & 0,26 & 76 & 0,35 & 0,33 \\
\hline 27 & 0,32 & 0,31 & 77 & 0,31 & 0,28 \\
\hline 28 & 0,52 & 0,52 & 78 & 0,19 & 0,19 \\
\hline 29 & 0,31 & 0,24 & 79 & 0,13 & 0,14 \\
\hline 30 & 0,19 & 0,18 & 80 & 0,83 & 0,81 \\
\hline 31 & 0,29 & 0,30 & 81 & 0,62 & 0,64 \\
\hline 32 & 0,22 & 0,23 & $8: 2$ & 0,72 & 0,75 \\
\hline 33 & 0,24 & $0,2 / x$ & 83 & 0,99 & 0,98 \\
\hline $3^{\prime} t$ & 0,25 & 0,25 & 84 & 1,09 & 1,04 \\
\hline 35 & 0,24 & 0,24 & 85 & 0,78 & 0,77 \\
\hline 36 & 0,32 & 0,29 & 86 & 0,74 & 0,72 \\
\hline 37 & 0,20 & 0,22 & 87 & 0,50 & 0,52 \\
\hline 38 & 0,32 & 0,31 & 88 & 0,72 & 0,70 \\
\hline 39 & 0,35 & $0,3^{\prime}$ & 89 & 1,15 & 1,13 \\
\hline 40 & 0,3 ' & $0,3^{\prime}$ & 90 & 1,50 & 1,69 \\
\hline 41 & 0,30 & 0,30 & 91 & 1,20 & 1,18 \\
\hline ' 2 & 0,29 & 0,31 & $92-3$ & 1,35 & 1,30 \\
\hline 43 & 0,28 & 0,30 & 93 & 0,35 & 0,40 \\
\hline 44 & 0,16 & 0,46 & 9 ' & 0,30 & 0,35 \\
\hline 15 & 0,33 & 0,32 & 95 & 0,48 & 0,48 \\
\hline 46 & 0,35 & 0,31 & 96 & 0,55 & 0,55 \\
\hline 47 & 0,19 & 0,23 & 97 & 0,60 & 0,57 \\
\hline 48 & 0,31 & 0,32 & 98 & 0,80 & 0,75 \\
\hline 49 & - & - & 99 & 0,80 & 0,81 \\
\hline 50 & 0,29 & 0,32 & 100 & 0,88 & 0,93 \\
\hline
\end{tabular}


Autrement dit, les deux méthodes donnent des résultats semblables.

Indiquons que la corrélation entre les cleux méthodes est excellente $(r: 0,996)$; nous avons tracé, de plus, la droite de régression $\mathrm{Y}=0,0 \mathrm{Io}+0,96 \mathrm{X}$ proche de $\mathrm{Y}=\mathrm{X}$.

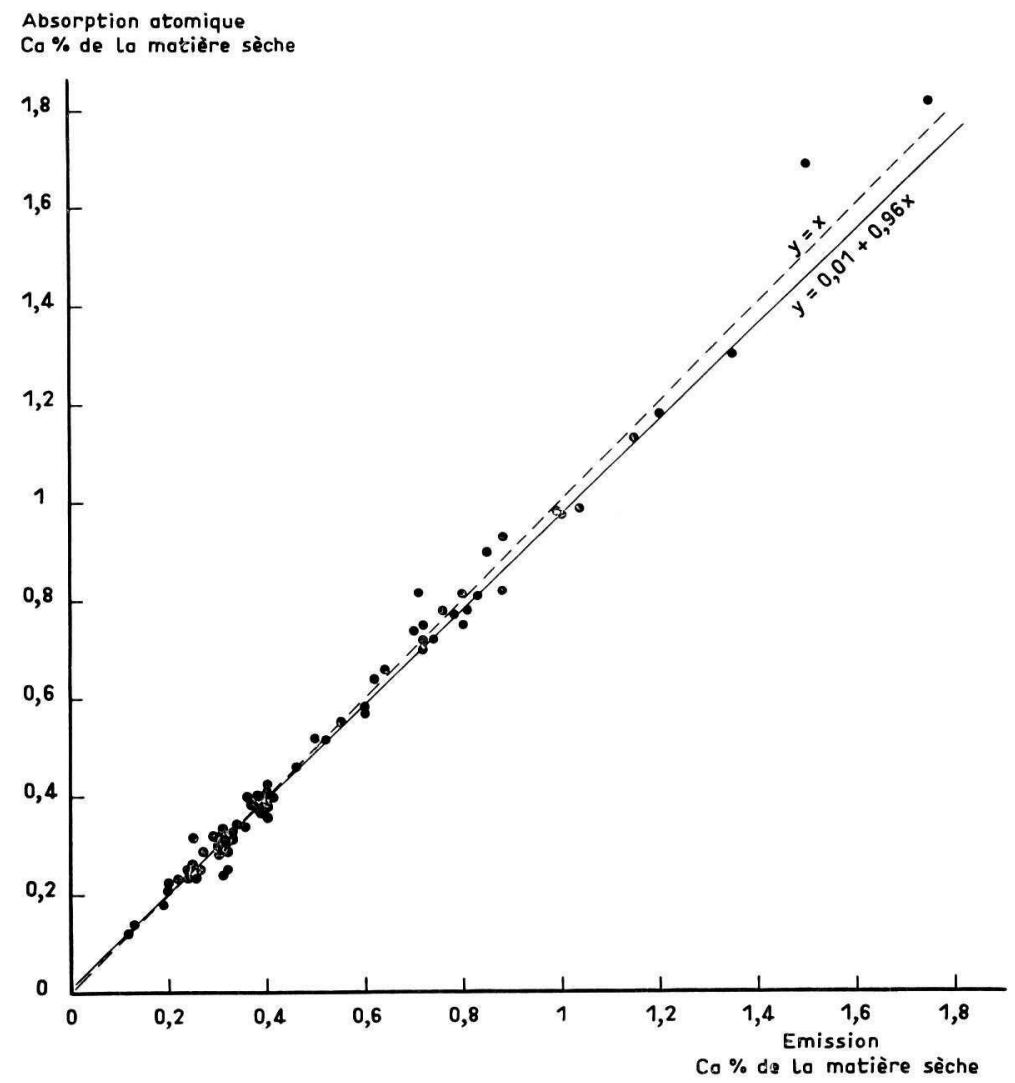

FIG. 2. - Droite de régression de Y (résultat par absorption atomique) en fonction de $X$ (résultat par émission)

Fig. 2. - Regression line of $Y$ (data obtained by atomic absorption) on $X$ (data obtained by emission)

\section{CONCLUSION}

Cette étude rapide montre qu'il est possible d'utiliser indifféremment la spectrométrie d'émission ou la spectrométrie d'absorption atomique pour doser le calcium dans les végétaux à condition d'évaluer par abaque l'interférence du phosphore dans le premier cas ou bien de réduire cette interférence par adjonction de chlorure de strontium, dans le second cas.

L'évaluation de l'interférence phosphore ne pose pas a priori de travail supplémentaire dans la mesure où la teneur en phosphore de ces échantillons foliaires est toujours demandée.

Remarquons que lorsque l'échantillon est très riche en phosphore l'interférence tend à devenir 
plus constante lorsque varie la teneur en calcium des échantillons. De ce fait, il est possible de pratiquer dans chaque échantillon un ajout de phosphore pour obtenir une interférence relativement constante. La précision des résultats est cependant moins bonne.

Rę̧u pour publication en juillet 1975.

\section{SUMMARY}

\section{COMPARAISON OF TWO CALCIUM ANALYTICAL, SPECTROMETRIC METHODS. EFFECT OF PHOSPHORUS INTERFERENCE}

Calcium determination in foliar tissues obtained by two spectrometric methods are compared (Atomic absorption spectrometry and Emission spectrometry).

Phosphorus interference with calcium in the emission spectrometry method has been measured. The two methods are equivalent if we take into account the inference corrections for the emission spectrometry method and add strontium chlorure for the determination with the atomic absorption method.

\section{RÉFÉRENCES BIBLIOGRAPHIQUES}

Charlot G,, I 6r. Les méthodes de la chimie analytique. Masson, ro24 p.

Clément A., I97r. Principes et méthodes d'analyses des macroéléments et microéléments dans les échantillons foliaires. 32 p. (Tirage intérieur).

Pinta M., et al. Ig7r. Spectrométrie d'absorption atomique. 2 tomes, Masson, 793 p.

Schwartz D., I963. Méthodes statistiques à l'usage des médecins et des biologistes. Flammarion. 